\title{
Análisis de características estereotípicas de género en líderes y seguidores
}

\section{Analysis of gender stereotypic characteristics in leaders and subordinates}

\author{
María Laura Lupano Perugini ${ }^{1} \&$ Alejandro Castro Solano \\ Consejo Nacional de Investigaciones Científicas y Técnicas \\ Universidad de Palermo
}

(Recepción: Abril 2010 - Aceptación: Octubre 2010)

\begin{abstract}
Resumen
Se realizó una investigación con el fin de analizar como se describen líderes y seguidores en función de las características estereotípicas de género percibidas y, además, verificar si existen diferencias en dichas características según tipo de puesto (líder/ seguidor). Participaron 612 sujetos adultos, 329 varones (54\%) y 283 mujeres $(46 \%)$, (media edad $=37,54$ años; $D E=11,88)$. El 59\% ocupaba puestos de dirección y el resto eran subordinados. Se utilizó una versión abreviada del Bem Sex Roles (Bem, 1974) para la recolección de los datos. Los resultados muestran que los líderes hombres se autodescriben principalmente con atributos agénticos-masculinos (e.g. dominante), comparado con los seguidores que lo hacen a partir de algunas características comunales-femeninas (e.g. sumisión). En relación a las mujeres, las líderes se autodescriben principalmente con atributos andróginos combinado cualidades de ambos géneros (e.g. dominante y sensible a las necesidades de otros); en cambio las empleadas destacaron atributos comunales (e.g. sumisón).

Palabras claves: características estereotípicas, género, líder, seguidor.
\end{abstract}

\begin{abstract}
An investigation was realized to analyze the way leaders and subordinates describe themselves in relation to perceived gender stereotypic characteristics and, to verify if exists differences in these characteristics according position (leader/ subordinate). Participate 612 individuals, 329 male (54\%) y 283 female (46\%), age average $=37,54$ years $(D E=11,88) .59 \%$ of the participants occupied executive positions and the rest were subordinates. It was utilized and abbreviate version of the Bem Sex Roles (Bem, 1974) to data recollection. The obtained results show that male leaders describe themselves mainly with agentic-masculine attributes (e.g. dominant), compared with subordinates who describe themselves with communal- feminine characteristics (e.g. submission). On the other hand, the female leaders describe themselves mainly with androgyny attributes combining qualities of both genders (e.g. dominant and sensible to the needs of others); however the female subordinates show communal attributes (e.g. submission).
\end{abstract}

Key words: stereotypic characteristics, gender, leader, subordinate.

\section{Introducción}

El estudio de las diferencias de género resulta relevante en relación a determinados fenómenos sociales, en este caso el liderazgo. La existencia de diferencias en el papel sexual y sus correlatos ha sido bastamente reconocida y documentada por los científicos sociales (Diaz-Loving, Rivera Aragón \& Sánchez Aragón, 2001). En el segundo cuarto del siglo XX, dentro de la psicología, apareció una

1 Correspondencia dirigida a: María Laura Perugini, Universidad de Palermo, Av Orrego 1279 (C.P. 1414), Buenos Aires, Argentina. E-Mail: mllupano@hotmail.com. 
manifiesta preocupación por responder empíricamente a la pregunta sobre el significado de la masculinidad y la feminidad. De ahí que fueran varios los instrumentos elaborados a tal fin (Fernández, Quiroga, Del Olmo \& Rodríguez, 2007; Vergara \& Páez, 1993).

Existen diferentes modelos teóricos que intentan explicar las diferencias de género. Parson y Bales en 1955 identificaron dos diferentes características: un papel instrumental orientado a metas que se asigna a los hombres en la mayor parte de las sociedades, y un papel expresivo orientado a las relaciones interpersonales que se asigna a las mujeres. En la misma línea, Bakan (1966) distingue como más común en los hombres la diligencia caracterizada por la autoafirmación y la autoprotección, y en las mujeres la comunión caracterizada por la abnegación y la preocupación por los demás. Bakan refiere que ambas dimensiones son separadas pero coexistentes en la personalidad de los sujetos, quedando supeditada a los procesos de socialización la conformación de características distintivas de hombres y mujeres. Tal como sostiene Diaz-Loving, Rivera Aragón y Sánchez Aragón (2001), los estudios que se realizan sobre esta temática suelen mostrar la importancia que tiene el contexto ecosistémico y sociocultural particular, en el que las prácticas de socialización generalmente están encaminadas a la transmisión de aspectos instrumentales en los hombres y expresivos en las mujeres.

Lo mencionado se relaciona con la conformación de estereotipos. Un estereotipo es una generalización que se realiza sobre una persona en razón de su pertenencia a un grupo o a una categoría social determinada. Más específicamente, un estereotipo de género refiere a un conjunto de creencias compartidas socialmente acerca de las características que poseen hombres y mujeres (Ashmore, Del Boca \& Wohlers, 1986; Bem, 1974; Eagly \& Karau, 2002; Moya 2003). En virtud de que se trata de construcciones sociales, cualquier cambio o transformación en las distintas sociedades puede conllevar a un cambio en el significado de dichas construcciones (Robinson, Shaver \& Wrigshtman, 1991). Por ejemplo, un meta-análisis de estudios realizados con escalas de masculinidad - feminidad en muestras norteamericanas entre 1970 y 1995 ha encontrado un aumento en la escala de masculinidad pero pocos cambios en la de feminidad tanto en hombres como en mujeres, registrándose el mayor aumento en la escala de masculinidad en el grupo de las mujeres (Twenge, 1997).

La conceptualización y definición teórica de la masculinidad y feminidad ha desembocado en diversas operacionalizaciones, inventarios o instrumentos (e.g. Bem, 1981; Spence \& Helmreich, 1978). Uno de los trabajos determinantes fue el desarrollado por Bem (1974) ya que marcó una división importante al diseñar el primer instrumento que evalúa estas dimensiones como medidas independientes (Bem Sex Roles Inventory- BSRI). Ella destacó que las personas pueden ser masculinas, femeninas o andróginas, éstas últimas presentan ambas características en forma balanceada. El BSRI es una de las cinco técnicas más usadas según el Mental Measurement Yearbook (Mitchel, 1985). Por su parte, Spence y Helmreich $(1974,1978)$ diseñaron el Personal Attributes Questionnaire (PAQ) que mide masculinidad y feminidad como dimensiones ortogonales. Otras técnicas diseñadas para medir estas dimensiones son: Personality Research Form - ANDRO (Berzins, Welling \& Better, 1978); Adjective Check List - ACL (Heilburn, 1976); Sex Role Behavior Scale (Orlofsky, 1981). Los mencionados instrumentos, en su mayor parte, coinciden en que parten de un modelo dualístico (masculinidad- feminidad como dimensiones independientes) que posibilita una cuádruple tipología (sujetos andróginos, masculinos, femeninos e indiferenciados) no relacionada con el dimorfismo sexual sino con la construcción social del género (Bem, 1974; Spence \& Helmreich, 1978; Spence \& Buckner, 2000).

Los antecedentes expuestos en relación a las diferencias de género resultan de fundamental importancia a la hora de explicar las desigualdades presentes en la mayoría de las sociedades occidentales en cuanto al acceso a puestos de dirección en distintos ámbitos y organizaciones ya que dicho acceso resulta menor para el caso de las mujeres (Cuadrado, 2004). La diferencia a favor de los hombres en dicho acceso se la ha explicado a través de la metáfora del Techo de Cristal (Federal Glass Ceiling Commission, 1995; Morrison, White \& Van Velsor, 1987) que intenta mostrar que existen barreras invisibles, pero efectivas, que permiten a las mujeres avanzar solo hasta un determinado nivel en la escala jerárquica de las organizaciones. Eagly y Carli (2007) sostienen que esta situación ha empezado a cambiar y que las mujeres presentan mayor acceso, piénsese, por ejemplo, en los cargos políticos presidenciales ocupados por mujeres en los últimos tiempos. Estas autoras proponen 
el concepto de laberinto como nueva metáfora en reemplazo de la ya mencionada, destacando que, en la actualidad, a las mujeres no les está bloqueado el acceso a los puestos más altos, pero sí deben sortear diferentes obstáculos y recorrer dificultosos caminos para poder llegar a ellos.

Tradicionalmente se ha asociado al liderazgo con el género masculino. Los cargos directivos suelen ser caracterizados con rasgos instrumentales/ agénticos, generalmente atribuidos a los hombres, como competitividad, control, autoridad u orientación hacia la tarea. Estas consideraciones hacen que características propiamente femeninas como la orientación y preocupación por los otros -rasgos expresivos/comunales- no sean consideradas esperables para los puestos de liderazgo, fomentando sesgos contra las mujeres en selección, promoción y ocupación de puestos directivos (Cuadrado, 2004; Eagly 1987).

En esta línea, la Teoría de congruencia del rol hacia las mujeres líderes, propuesta por Eagly y Karau (2002), defiende que el prejuicio hacia las mujeres líderes es una de las causas de las dificultades que presentan las mujeres para acceder a puestos de liderazgo. El prejuicio procedería de la incongruencia entre las características femeninas y los requisitos del rol de líder. El constructo clave de la Teoría es el de roles de género, es decir, aquellas creencias compartidas acerca de los lugares sociales ocupados por hombres y mujeres (Morales \& Cuadrado, 2004). Los roles incluyen dos tipos de expectativas o normas: descriptivas -expectativas compartidas sobre lo que los miembros de un grupo realmente hacen-y prescriptivas -expectativas compartidas sobre lo que los miembros de un grupo idealmente deberían hacer.

En términos generales, las personas tienden a creer que para desempeñarse efectivamente en puestos de liderazgo es necesario desplegar cualidades masculinas, principalmente en roles definidos de modo más agéntico (e.g. roles militares). Dicha percepción ha presionado a muchas mujeres a adoptar características similares a los hombres. Sin embargo, cuando algunas mujeres llegan a ser consideradas igual de competentes que sus pares varones, las personas tienden a considerar que violan las normas esperadas socialmente (o prescriptivas) y reaccionan negativamente frente a ellas (Butler \& Geis, 1990). Por ende, las mujeres líderes pueden llegar a ser evaluadas negativamente por dos razones: ya sea porque no despliegan las características que suelen relacionarse con el liderazgo efectivo o porque, en el caso de que las desplieguen, son consideradas poco femeninas. De ambas formas existe incongruencia entre el rol de liderazgo y el rol social. En consecuencia se ven obligadas a combinar atributos asociados al estereotipo masculino (asertividad, competencia, control) con cualidades femeninas, con el fin de lograr no ser rechazadas principalmente por sus pares varones.

De lo antedicho se desprende la relevancia de realizar trabajos que aborden las diferencias en la autoasignación de características estereotípicas de género en hombres y mujeres, tanto líderes como seguidores. Cuadrado (2004) destaca la escasez de trabajos en esta línea (Veáse Rosener, 1990; López-Zafra \& Del Olmo, 1999; Singh, 1994). En un estudio realizado por la mencionada autora se halló que los hombres y las mujeres líderes se describían de manera similar tanto en los atributos instrumentales como comunales. Sin embargo, al analizar una muestra de subordinados se encontró que las mujeres se autoasignan con mayor intensidad características comunales y los hombres características instrumentales. Por lo tanto, parece ser que los hombres y mujeres que no ocupan puestos de liderazgo tienen interiorizados sus respectivos estereotipos de género. Por su parte, las mujeres líderes se diferencian de las seguidoras en la mayor autoasignación de características instrumentales (Cuadrado, 2004; Cuadrado, Navas \& Molero, 2006).

Cabe destacar que, en investigaciones realizadas con muestras conformadas por mujeres y hombres que no ocupan necesariamente posiciones de liderazgo, si bien se constata un mantenimiento de la de tradición que inculca preferencialmente en los hombres atributos instrumentales y en las mujeres atributos expresivos, también se evidencia una mayor asignación a las mujeres de características como la responsabilidad, el orden o el ser mas trabajadoras, la cuales constituyen características típicamente masculinas (Diaz-Loving, Rivera Aragón \& Sánchez Aragón, 2001; Diaz-Loving, Rocha Sánchez \& Rivera Aragón, 2004).

Por último, resulta relevante subrayar que se han realizado muchos estudios con instrumentos como el BSRI con el fin de identificar la imagen del líder ideal. Si bien la mayoría de las investigaciones han 
mostrado que esa figura estaría compuesta por rasgos estereotípicos masculinos (e.g. Barberá, Ramos \& Sarrió, 2000; Monroy, 1994; Osca \& López-Sáez, 1994), muchos estudios están dando cuenta que, últimamente, las organizaciones demandan de sus líderes características comunales en combinación con las instrumentales (e.g. Cuadrado, Navas \& Molero, 2006; Pérez \& Morales, 1995).

En función de lo previamente mencionado, el presente estudio tiene los siguientes objetivos:

1. Analizar como se describen líderes y seguidores varones y mujeres en función de las características estereotípicas de género percibidas.

2. Analizar si existen diferencias en las características estereotípicas de género percibidas según tipo de puesto (líder vs. seguidor) para el grupo de los varones y para el grupo de las mujeres.

\section{Método}

\section{Participantes}

Participaron, de forma voluntaria, 612 sujetos adultos, 329 varones (54\%) y 283 mujeres (46\%) que tenían en promedio 37,54 años $(D E=11,88)$. El 59\% $(n=361)$ de los participantes ocupaba puestos de dirección o gerenciamiento, y el 41\% $(n=251)$ restante eran subordinados. El 23,7\% $(n$ $=145)$ trabajaba en empresas pequeñas, el $42,3 \%$ se desempeñaba en empresas medianas $(n=259)$ y el restante $32,7 \%$ se desempeñaba en grandes empresas $(n=200)$. De acuerdo al cargo desempeñado se definieron cinco grupos, de mayor a menor, con respecto a la categoría ocupacional. El $32,2 \%$ eran funcionarios, directivos y/o profesionales -se trataba de la categoría ocupacional más alta- $(n=197)$. El $26,3 \%$ eran jefes de nivel intermedio $(n=161)$. El 34,3\% $(n=210)$ trabajaba como comerciantes, empleados calificados, docentes, entre otros. El 4,4\% $(n=27)$ trabajaba como cuentapropistas, trabajadores de servicios, choferes, empleados no calificados, entre otros. E1 2,8\% restante se dedicaba a actividades no incluidas en las categorías precedentes $(n=17)$.

Con respecto al nivel educativo, la mayoría $(76,7 \%)$ estaba cursando o poseía estudios universitarios o superiores $(n=470)$. El 14,2\% $(n=87)$ refería estar cursando o haber finalizado estudios terciarios; y el 9,1\% restante presentaba un nivel de estudios menor -principalmente secundario completo- $(n=55)$.

La mayor parte de los participantes $(88,1 \% ; \mathrm{n}=539)$ residían en la ciudad de Buenos Aires y el conurbano bonaerense (Argentina). El resto $(11,6 \%)$ se distribuía en distintas regiones del país, y solo el $0,3 \%(n=2)$ residía en el exterior.

\section{Instrumentos}

\section{Inventario de Roles Sexuales-Bem Sex Roles/BSRI-}

Se utilizó una versión abreviada y en castellano del Bem Sex Roles (Bem, 1974), el instrumento cuenta con adecuadas propiedades psicométricas (Ver Fernández, 2001; López-Sáez \& Morales, 1995). Este inventario permite categorizar a los sujetos en base al rol sexual en función de las características intrumentales/ agénticas/ masculinas (e.g. dominante, agresivo, individualista) y las expresivas/ comunales/ femeninas (e.g. comprensivo, compasivo, cálido). Consiste en un listado de 18 adjetivos con formato Likert de respuesta con 7 opciones (1 a 7). Presenta dos dimensiones: Masculinidad-instrumentalidad y Feminidad-expresividad, de nueve ítems cada una. El sujeto debe marcar el grado en el que considera que el adjetivo lo describe. Para la muestra local, se calculó la fiabilidad para cada dimensión utilizando el coeficiente alpha de Cronbach (consistencia interna). Para la dimensión masculinidad - instrumentalidad se obtuvo un alpha de 0,76 y, para la dimensión feminidad - expresividad, de 0,81 . Los citados coeficientes se encuentran dentro de valores aceptables.

A partir de los datos muestrales, el instrumento permite clasificar a los sujetos evaluados en cuatro categorías: 
Femenino: refiere a sujetos que se describen con atributos típicamente femeninos o comunales. Se clasifican en esta categoría aquellos sujetos que obtienen valores superiores a la mediana muestral en la escala de feminidad- expresividad y valores inferiores a la mediana en la escala masculinidad- instrumentalidad.

Masculino: refiere a sujetos que se describen con atributos típicamente masculinos o agénticos. Se clasifican en esta categoría aquellos sujetos que obtienen valores superiores a la mediana muestral en la escala de masculinidad- instrumentalidad y valores inferiores a la mediana en la escala feminidad- expresividad.

Andrógino: refiere a sujetos que se describen con atributos tanto comunales como agénticos (es decir, de ambos géneros). Se clasifican en esta categoría aquellos sujetos que obtienen valores superiores a la mediana muestral tanto en la escala de masculinidad- instrumentalidad como en la de feminidad- expresividad.

Indiferenciado: refiere a sujetos que se describen débilmente con atributos de ambos géneros. Se clasifican en esta categoría aquellos sujetos que obtienen valores inferiores a la mediana muestral tanto en la escala de masculinidad- instrumentalidad como en la de feminidad- expresividad.

Este método de división por la mediana fue propuesto primero por Spence y col. en 1975 y luego adoptado por Bem (1981) y resulta ser el procedimiento más usado (Vergara \& Páez, 1993; Lenney, 1981; Archer, 198).

\section{Procedimiento}

Los instrumentos formaban parte de una batería de pruebas más amplia que se administró con el propósito de obtener información acerca de teorías implícitas, creencias y actitudes acerca del liderazgo femenino y masculino. Las tareas de recolección y entrada de datos estuvieron a cargo de un becario. El análisis de los datos se efectuó con el procesador SPSS versión 13.0.

El estudio es descriptivo - correlacional (de diferencia de grupos), con diseño transversal.

\section{Resultados}

Descripción de líderes y seguidores varones y mujeres en función de las características estereotípicas de género percibidas.

Con el fin de responder al objetivo número 1 que intenta describir a líderes y seguidores varones y mujeres en función de las características estereotípicas de género, se estimó la mediana muestral para ambas dimensiones (Masculinidad- instrumentalidad y Feminidad- expresividad) obteniéndose los valores que se muestran en la Tabla 1 .

Tabla 1. Medianas y desvíos muestrales para las escalas del BSRI

\begin{tabular}{llcc}
\hline Sexo & Descriptivos & $\begin{array}{c}\text { Escala Masculinidad- } \\
\text { instrumentalidad }\end{array}$ & $\begin{array}{c}\text { Escala Feminidad - expre- } \\
\text { sividad }\end{array}$ \\
\hline HOMBRE & $N$ & 329 & 329 \\
& Mediana & 39,00 & 39,00 \\
\multirow{3}{*}{ MUJER } & DE & 8,02 & 8,06 \\
& $N$ & 283 & 283 \\
& Mediana & 35,00 & 46,00 \\
TOTAL & DE & 8,27 & 7,43 \\
& $N$ & 612 & 612 \\
& Mediana & 37,00 & 42,00 \\
& DE & 8,38 & 8,36 \\
\hline
\end{tabular}


En función de las medianas obtenidas para hombres y mujeres y el sistema de clasificación propuesto para el BSRI (Ver Instrumentos) se obtuvo la siguiente clasificación teniendo en cuenta el sexo y tipo de puesto (líder/ seguidor):

Tabla 2. Clasificación del BSRI según sexo y tipo de puesto (líder/ seguidor)

\begin{tabular}{cccccc}
\hline Sexo & Puesto & Masculino & Femenino & Andrógino & Indiferenciado \\
\hline HOMBRE & Líder & $\mathbf{3 3 , 8} \%$ & $22,4 \%$ & $26,9 \%$ & $16,9 \%$ \\
& $(n=201)$ & $(n=68)$ & $(n=45)$ & $(n=54)$ & $(n=34)$ \\
& Seguidor & $19,6 \%$ & $\mathbf{3 2 , 8 \%}$ & $21,9 \%$ & $25,8 \%$ \\
& $(n=128)$ & $(n=25)$ & $(n=42)$ & $(n=28)$ & $(n=33)$ \\
& Líder & $26,3 \%$ & $35,0 \%$ & $35,6 \%$ & $3,1 \%$ \\
& $(n=160)$ & $(n=42)$ & $(n=56)$ & $(n=57)$ & $(n=5)$ \\
& Seguidor & $26,0 \%$ & $\mathbf{4 1 , 5 \%}$ & $15,4 \%$ & $17,1 \%$ \\
& $(n=123)$ & $(n=32)$ & $(n=51)$ & $(n=19)$ & $(n=21)$ \\
\hline
\end{tabular}

Como se puede observar en la Tabla 2, los líderes hombres se autodescriben con atributos típicos de su género, seguido de características andróginas; en tanto que los seguidores lo hacen con características principalmente femeninas y, en segundo lugar, de forma indiferenciada.

En el caso de las mujeres, las líderes se autodescriben con atributos andróginos en primer lugar seguido de los femeninos. Esto destaca la particularidad de la combinación de propiedades de ambos géneros para convertirse en líderes efectivas. Resulta relevante el bajo porcentaje de mujeres líderes que se describen de modo indiferenciado mostrando que este grupo parece tener bien definidas sus cualidades. En cambio, las empleadas se describen con propiedades femeninas, quedando las otras categorías bastante alejadas.

Diferencias en las características estereotípicas de género percibidas según tipo de puesto (líder vs. seguidor) para el grupo de los varones.

Como parte del segundo objetivo se intentó verificar si existen diferencias en las características estereotípicas de género percibidas según tipo de puesto (líder vs. seguidor) para el grupo de los varones.

Con tal fin, se realizaron análisis de varianza univariante para cada atributo y las dimensiones. Se estimaron también los índices de tamaño del efecto eta cuadrado que indican la proporción de varianza explicada por cada fuente de variación. Se hallaron diferencias significativas en la dimensión instrumentalidad y algunas características tal como se muestran en la Tabla 3: 
Tabla 3. Diferencias en las características estereotípicas de género percibidas según tipo de puesto (líder/ seguidor) para el grupo de los varones $(n=329)$

\begin{tabular}{|c|c|c|c|c|}
\hline \multirow[b]{2}{*}{ Característica } & \multicolumn{2}{|c|}{ Media $(D E)$} & \multirow[t]{2}{*}{$F$} & \multirow[t]{2}{*}{$\eta^{2}$} \\
\hline & $\begin{array}{c}\text { Líder } \\
(n=201)\end{array}$ & $\begin{array}{l}\text { Seguidor } \\
(n=128)\end{array}$ & & \\
\hline 1. Atlético/a, deportivo/a & $4,57(1,68)$ & $4,58(1,55)$ & $0,00 n s$ & 0,00 \\
\hline 2. Cariñoso/a & $4,66(1,47)$ & $4,77(1,29)$ & $0,48 n s$ & 0,00 \\
\hline 3. Personalidad fuerte & $5,45(1,16)$ & $4,9(1,18)$ & $17,16^{* *}$ & 0,05 \\
\hline $\begin{array}{l}\text { 4. Sensible a las necesidades } \\
\text { de los demás }\end{array}$ & $5,20(1,33)$ & $5,30(1,14)$ & $0,44 n s$ & 0,00 \\
\hline $\begin{array}{l}\text { 5. Desea arriesgarse, amante } \\
\text { del peligro }\end{array}$ & $4,48(1,68)$ & $4,53(1,65)$ & $0,05 n s$ & 0,00 \\
\hline 6. Comprensivo/a & $5,21(1,28)$ & $5,11(1,11)$ & $0,56 n s$ & 0,00 \\
\hline 7. Compasivo/a & $4,68(1,43)$ & $4,72(1,26)$ & $0,07 n s$ & 0,00 \\
\hline 8. Dominante & $5,05(1,39)$ & $4,09(1,5)$ & $35,46 * *$ & 0,09 \\
\hline 9. Cálido/a, afectuoso/a & $4,72(1,47)$ & $5,05(1,14)$ & $4,51^{*}$ & 0,01 \\
\hline 10. Tierno/a, delicado/a, suave & $3,87(1,6)$ & $4,27(1,53)$ & $4,98^{*}$ & 0,01 \\
\hline 11. Agresivo/a, combativo/a & $4,04(1,57)$ & $3,83(1,63)$ & $1,45 n s$ & 0,00 \\
\hline 12. Actúa como líder & $5,62(1,3)$ & $4,67(1,5)$ & $39,74 * *$ & 0,10 \\
\hline 13. Individualista & $4,17(1,81)$ & $3,44(1,52)$ & $14,17 * *$ & 0,04 \\
\hline 14. Amante de los niños & $4,8(1,49)$ & $4,94(1,55)$ & $0,7 n s$ & 0,00 \\
\hline 15. Llora fácilmente & $2,6(1,49)$ & $2,83(1,72)$ & $1,74 n s$ & 0,00 \\
\hline 16. Duro/a & $4,26(1,5)$ & $3,69(1,45)$ & $11,57 * *$ & 0,03 \\
\hline 17. Sumiso/a & $2,46(1,41)$ & $2,79(1,41)$ & $4,23 *$ & 0,01 \\
\hline 18. Egoísta & $2,96(1,65)$ & $2,73(1,55)$ & $1,48 n s$ & 0,00 \\
\hline Dimensión Instrumentalidad & $40,61(7,64)$ & $36,46(7,83)$ & $22,18 * *$ & 0,06 \\
\hline Dimensión Expresividad & $38,21(8,26)$ & $39,79(7,67)$ & $3 n s$ & 0,00 \\
\hline
\end{tabular}

$* * p<0.01$

$* p<0.05$

Como puede observarse en la Tabla 3, las diferencias entre líderes y seguidores hombres reflejan una mayor cantidad de atributos agénticos (personalidad fuerte, dominante, actúa como líder, individualista y duro) en los líderes, en comparación con los seguidores, quienes han evidenciado una diferencia a favor del rasgo de sumisión considerado una característica comunal. Debe tenerse en cuenta que los tamaños de efecto observados son bajos, limitando la significación práctica de las 
diferencias encontradas. Se obtuvo el tamaño más alto en el atributo actúa como líder que explica un $10 \%$ de la varianza encontrada, seguido de la característica dominante que explica un $9 \%$.

Diferencias en las características estereotípicas de género percibidas según tipo de puesto (líder vs. seguidor) para el grupo de las mujeres

Para responder a la segunda parte del segundo objetivo, se trabajó con los datos correspondientes a las mujeres realizándose análisis de varianza univariante para cada atributo y las dimensiones. Se estimaron también los índices de tamaño del efecto eta cuadrado. Se hallaron diferencias significativas en la dimensión instrumentalidad y algunas características tal como se muestran en la Tabla 4:

Tabla 4. Diferencias en las características estereotípicas de género percibidas según tipo de puesto (líder/ seguidor) para el grupo de las mujeres $(\mathrm{n}=283)$

\begin{tabular}{|c|c|c|c|c|}
\hline \multirow{3}{*}{ Característica } & \multicolumn{2}{|c|}{ Media (DE) } & \multirow[t]{3}{*}{$F$} & \multirow[t]{3}{*}{$\eta^{2}$} \\
\hline & Líder & Seguidor & & \\
\hline & $(n=160)$ & $(n=123)$ & & \\
\hline 1. Atlético/a, deportivo/a & $3,47(1,85)$ & $3,51(1,75)$ & $0,04 n s$ & 0,00 \\
\hline 2. Cariñoso/a & $5,41(1,25)$ & $5,39(1,25)$ & $0,02 n s$ & 0,00 \\
\hline 3. Personalidad fuerte & $5,61(1,21)$ & $5(1,36)$ & $15,53 * *$ & 0,05 \\
\hline $\begin{array}{l}\text { 4. Sensible a las necesidades } \\
\text { de los demás }\end{array}$ & $5,96(0,97)$ & $5,67(1,17)$ & $5,04 *$ & 0,01 \\
\hline $\begin{array}{l}\text { 5. Desea arriesgarse, amante } \\
\text { del peligro }\end{array}$ & $4,02(1,68)$ & $3,61(1,71)$ & $4,05 *$ & 0,01 \\
\hline 6. Comprensivo/a & $5,74(1,06)$ & $5,53(1,07)$ & $2,65 n s$ & 0,00 \\
\hline 7. Compasivo/a & $5,36(1,31)$ & $5,03(1,28)$ & $4,33 *$ & 0,01 \\
\hline 8. Dominante & $4,83(1,46)$ & $4,19(1,58)$ & $12,53 * *$ & 0,04 \\
\hline 9. Cálido/a, afectuoso/a & $5,67(1,24)$ & $5,54(1,14)$ & $0,74 n s$ & 0,00 \\
\hline $\begin{array}{l}\text { 10. Tierno/a, delicado/a, } \\
\text { suave }\end{array}$ & $4,91(1,37)$ & $4,93(1,46)$ & $0,01 n s$ & 0,00 \\
\hline 11. Agresivo/a, combativo/a & $3,61(1,6)$ & $3,41(1,6)$ & $1,14 n s$ & 0,00 \\
\hline 12. Actúa como líder & $5,21(1,34)$ & $4,12(1,65)$ & $37,60 * *$ & 0,11 \\
\hline 13. Individualista & $3,54(1,68)$ & $3,18(1,73)$ & $3,18 n s$ & 0,00 \\
\hline 14. Amante de los niños & $5,41(1,55)$ & $5,26(1,69)$ & $0,57 n s$ & 0,00 \\
\hline 15. Llora fácilmente & $4,07(1,91)$ & $4,23(1,97)$ & $0,46 n s$ & 0,00 \\
\hline 16. Duro/a & $3,79(1,56)$ & $3,23(1,48)$ & $9,36^{* *}$ & 0,03 \\
\hline 17. Sumiso/a & $2,72(1,46)$ & $3,14(1,5)$ & $5,59 *$ & 0,02 \\
\hline 18. Egoísta & $2,45(1,49)$ & $2,52(1,4)$ & $0,16 n s$ & 0,00 \\
\hline Dimensión Instrumentalidad & $36,53(8,02)$ & $32,76(8,12)$ & $15,16^{* *}$ & 0,05 \\
\hline Dimensión Expresividad & $45,24(7,19)$ & $44,72(7,74)$ & $0,33 n s$ & 0,00 \\
\hline
\end{tabular}


Como se observa en la Tabla 4, las empleadas mujeres presentan a su favor el mismo atributo que los empleados hombres: la sumisión, siendo esta una característica comunal que evidentemente diferencia a líderes y seguidores más allá del sexo. En términos generales, se evidencia diferencias a favor de las líderes mujeres en la dimensión instrumentalidad, pero si se analizan todos los atributos que generan discrepancia, se observa una combinación de características agénticas (personalidad fuerte, desea arriesgarse, dominante, actúa como líder y duro) junto con atributos expresivos (sensible a las necesidades de los demás y compasivo). Estos resultados son compatibles con resultados previos y con lo hallado en cuanto a la tipología en la que se ubican las mujeres líderes ya que se vio que en primer lugar se autodescriben como andróginas (Ver resultados objetivo 1).

Al igual que para el grupo de los hombres, debe tenerse en cuenta que los tamaños de efecto observados son bajos, limitando la significación práctica de las diferencias halladas. Se obtuvo el tamaño más alto en el atributo actúa como líder que explica un $11 \%$ de la varianza encontrada, seguido de la dimensión instrumentalidad y el atributo personalidad fuerte que explican el $5 \%$ de la varianza cada una.

\section{Discusión}

El presente trabajo se realizó con el fin de analizar y comparar como se describen líderes y seguidores varones y mujeres en función de las características estereotípicas de género percibidas. La realización de estudios locales resulta de fundamental importancia en virtud de que, como se mencionó previamente, la conceptualización de la masculinidad y la feminidad está culturalmente determinada, por lo que es esperable encontrar diferencias a través del tiempo y de los grupos culturales.

Los resultados a los que se arribó se encuentran en consonancia con hallazgos teóricos y empíricos previos.

Se pudo observar que los hombres líderes se autodescriben con atributos típicos de su género, los cuales a su vez son los que suelen relacionarse con el liderazgo efectivo (e.g. personalidad fuerte, dominante, actúa como líder, individualista y duro). En cambio, en el caso de las líderes mujeres se autodescribieron como andróginas, combinando características comunales y expresivas, por ejemplo se percibieron con personalidad fuerte y dominantes pero, a la vez, sensibles a las necesidades de los demás y compasivas. Por un lado, dichos resultados reflejan lo expuesto por Eagly y Karau (2002), en cuanto a que las mujeres líderes deben combinar ambos atributos con el fin de lograr no ser rechazadas principalmente por sus pares varones. Por otro lado, son congruentes con las investigaciones expuestas ya que aquellas también mostraban la creciente autoasignación de características instrumentales en el caso de las mujeres, principalmente las líderes. Sin embargo, en este trabajo se halló que tanto hombres como mujeres seguidoras se describen como femeninos/as asumiendo en mayor medida (comparado con los/las líderes) la característica de sumisión, esto parecería indicar que se trataría de una característica comunal que evidentemente diferencia a líderes y seguidores más allá del sexo. Estos resultados no son congruentes con los de Cuadrado (2004) que halló que los hombres y mujeres que no ocupaban puestos de liderazgo se describían asumiendo sus respectivos estereotipos de género.

Si se tiene en cuenta que los roles de liderazgo están empezando a sufrir transformaciones y, cada vez más, se privilegia la asunción de aspectos comunales orientados hacia los otros para liderar efectivamente, entonces la autodescripción que realizan las mujeres líderes puede resultar un aspecto favorecedor. En este sentido Eagly y Carli (2007) sostienen que si el rol de líder se ha hecho más femenino y las mujeres líderes más masculinas se podría haber logrado un estilo andrógino más apropiado. Lo antedicho reviste gran importancia ya que los mencionados cambios pueden aminorar la incongruencia percibida entre el rol de líder y el rol social femenino, que como fue expuesto se encuentra en la base del prejuicio hacia las mujeres líderes, principal obstáculo en el acceso a los puestos directivos. 
Si bien el análisis de las características de género permite explicar las dificultades que se le presentan a las mujeres en el acceso a posiciones de liderazgo, existen otras variables psicosociales no analizadas en este estudio que pueden estar relacionadas. Cuadrado (2002) menciona que variables como el contexto organizacional, los valores, el poder y las expectativas (además de las características de género) pueden proporcionar explicaciones más concluyentes que, por ejemplo, los estilos de liderazgo. Cabe aclarar, en relación a los estudios sobre estilos de liderazgo, que las investigaciones no han dado lugar a resultados concluyentes acerca de la existencia de diferentes estilos de liderar entre hombres y mujeres (Cuadrado \& Molero, 2002; Eagly \& Johnson, 1990; Eagly, JohannesenSchmidt \& Van Engen, 2003), de allí el interés en investigar otras posibles variables asociadas. Futuros estudios deberán abordar, a nivel local, la influencia de las variables psicosociales mencionadas en relación al fenómeno estudiado. También es importante que se estudie, no solo la autodescripción de los líderes sino la percepción de los seguidores sobre aquellos ya que el liderazgo es fundamentalmente un proceso atributivo resultado de un proceso de percepción social, siendo la esencia del mismo el ser percibido como líder por los otros (Lord \& Maher, 1991).

\section{Referencias}

Archer, J. (1989). The relationship between gender-role measmes: A review. British Journal of Social Psychology, 28, 173-184.

Ashmore, R. D.; Del Boca, F.K. \& Wohlers, A.J. (1986). Gender Stereotypes. En R.D. Ashmore \& F.K. Del Boca (Eds.), The Social Psychology of female-male relations: A critical analysis of central concepts (pp. 69-119). Orlando: Academic Press.

Bakan D. (1966). The duality of human existence. Chicago, C.A.: Rand McNally.

Barberá, E.; Ramos, A. \& Sarrió, M. (2000). Mujeres directivas ante el tercer milenio: el proyecto Norwdi XXI. Papeles del Psicólogo, 75, 46-52.

Bem S. (1974). The measurement of psychological androgyny. Journal of consulting and clinical psychology, 42, 155162.

Bem, S. (1981). Gender schema theory: a cognitive account of sex typing. Psychological Review , 88 (4), 354-364.

Berzins, J. I.; Welling, M. A. \& Better, R.E. (1978). A new measure of psychological androgyny based on the personality research form. Journal of consulting and clinical psychology, 46, 126-138.

Butler, D. \& Geis, F. L. (1990). Nonverbal affect responses to male and female leaders: Implications for leadership evaluations. Journal of Personality and Social Psychology, 58, 48-59.

Cuadrado, I. \& Molero, J.F. (2002). Liderazgo transformacional y género: autoevaluaciones de directivos y directivas españolas. Revista de Psicología de Trabajo y de las Organizaciones, 18 (1), 39-55.

Cuadrado, I. (2002). Estilos de liderazgo y género: Una perspectiva psicosocial. Tesis doctoral publicada en edición electrónica. ISBN: 84-9240-597-7. Almería: Servicio de Publicaciones de la Universidad de Almería.

Cuadrado, I. (2004). Valores y rasgos estereotípicos de género de mujeres líderes. Psicothema, 16 (2), 270- 275.

Cuadrado, I.; Navas, M. \& Molero, F. (2004). El acceso de las mujeres a puestos directivos: género, contexto organizacional y estilos de liderazgo. Rev. de Psicol. Gral. y Aplic., 57 (2), 181-192.

Diaz-Loving, R.; Rivera Aragón, S. \& Sánchez Aragón, R. (2001). Rasgos instrumentales (masculinos) y expresivos (femeninos), normativos (típicos e ideales) en México. Revista latinoamericana de Psicología, 33 (2), 131-139. 
Diaz-Loving, R.; Rocha Sánchez T. S. \& Rivera Aragón, S. (2004). Elaboración, validación y estandarización de un inventario para evaluar las dimensiones atributivas de instrumentalidad y expresividad. Revista Interamericana de Psicología, 38 (2), 263-276.

Eagly, A. H. (1987). Sex differences in social behaviour: A social- role interpretation. Hillsdale, NJ: Lawrence Erbaum.

Eagly, A. H. \& Karau, S. J. (2002). Role congruity theory of prejudice toward female leaders. Psychological Review, 109, 573- 598.

Eagly, A. H.\& Carli, L. (2007). Trough the labyrinth. The truth about how women become leaders. Boston: Harvard Business School Press.

Eagly, A. H. \& Johnson, B. T. (1990). Gender and leadership style: A meta-analysis. Psychological Bulletin, 108, 233-256.

Eagly, A. H.; Johannesen-Schmidt, M. C. \& Van Engen, M. L. (2003). Transfromational, Transactional, and Laissez- Faire Leadership Styles: A Meta- Analysis Comparing Women and Men. Psychological Bulletin, 129, 569- 591.

Federal Glass Ceiling Commission (1995). Good for business: Making full use of the nation's human capital: The environment scan: A fact finding report of the Federal Glass Ceiling Commission. Washington, DC: U.S. Government Printing Office.

Fernández, J.; Quiroga, M. A.; Del Olmo, I. \& Rodríguez, A. (2007). Escalas de masculinidad y feminidad: estado actual de la cuestión. Psicothema, 19 (3), 357-365.

Heilburn, A. B. (1976). Measurement of masculine and feminine sex roles identities as independent dimensions. Journal of consulting and clinical psychology, 44, 183-190.

Lenney, L. (1991). Sex Roles: the measurement of masculinity, feminity and androgyny. En: J.P. Robinson, P.R Shaver \& L.S. Wrightsman (Eds.). Measures of personality and social psychological attitudes. New York, USA: Wrigh Brian Academic Press.

López-Sáez, M. \& Morales, J.F. (1995). Gender stereotyping in the Spanish population: Looking into the future. En L. Amancio \& C. Nogueira (Eds.), Gender, Management and Science (pp. 151-168). Braga: Instituto de Educacao e Psicología.

López-Zafra, E. \& Del Olmo, S.M. (1999). Estereotipia de género y liderazgo transformacional en contextos de trabajo típicamente femeninos. Revista de Psicología Social Aplicada, 9 (3), 53-71.

Lord, R. \& Maher, K. (1991). Leadership and information processing. London: Routledge.

Mitchell, J.V. Jr. (1985). The $9^{\circ}$ mental measurements yerarbook. Lincoln, NE,USA: The Buros Institute of Mental Measurement.

Monroy, M.T. (1994). Liderazgo femenino y cultura de la empresa. Antropológica, 15/16, 145-154.

Morales, J. F. \& Cuadrado, I. (2004). Introducción: Teoría de congruencia de rol del prejuicio hacia líderes femeninos. Rev. de Psicol. Gral. y Aplic., 57 (2), 135- 146.

Morrison, A. M.; White, R.P. \& Van Velsor, E. (1987). Breaking the glass ceiling: Can women reach the top of America's largest corporations? Reading, MA: Addison- Wesley.

Moya, M.C. (2003). El análisis psicosocial del género. En J.F. Morales \& C. Huici (Eds.). Estudios de Psicología Social (pp. 175-222). Madrid: UNED.

Orlofsky, J. (1981). Relationship between Sex Role Attitudes and Personality Traits and the Sex Role Behavior Scale: A new measure of masculine and feminine role behaviors and interests. Journal of Personality and Social Psychology, 40, 927-940. 
Osca, A. \& López-Sáez, M. (1994). Desarrollo de carrera y género. Factores que influyen en las diferencias entre hombres y mujeres. Psicología del Trabajo y de las Organizaciones, 10 (28), 73-85.

Parson T. \& Bales, R.F. (1955). Family, socialization and interaction process. New York: Free Press.

Perez, J.A. \& Morales, J. F. (1995). Factores psicosociales que inciden en el acceso de las mujeres a los puestos de dirección. Memoria de estudio subvencionado por el Instituto de la Mujer, convocatoria 7-5-92. Documento no publicado.

Robinson, J.; Shaver, P. \& Wrightsman, L.S. (1991). Measures of personality and social psychological attitudes. New York, USA: Wrigh Brian Academic Press.

Rosener, J. B. (1990). Ways women leads. Harvard Business Review, 68, 119-125.

Singh, S. (1994). Gender differences in work values and personality characteristic among Indian executives. The Journal of Social Psychology, 134 (5), 699-700.

Spence, J. \& Buckner, C. (2000). Instrumental and expressive traits, trait stereotypes and sexist attitude: What do they signify? Personality of Women Quarterly, 24, 44-62.

Spence, J. \& Helmreich, R. (1978). Masculinity and feminity: their psychological dimensions, correlatos and antecedents. Austin, TX: University of Texas Press.

Spence, J.; Helmreich, R. \& Stapp, J. (1974). The personal attributes questionnaire: a measure of sex roles stereotypes and masculinity-feminity. JSAS: Catalog of selected documents in psychology, 4, 43-44.

Twenge, J. (1997). Changes in masculine and feminine traits over time: A meta-analysis. Sex Roles, $36(5 / 6), 305-325$.

Vergara A.I. \& Páez, D. (1993). Revisión teórico metodológica de los instrumentos para la medición de la identidad de género. Revista de Psicología Social, 8 (2), 133-152. 